\title{
The Wave Packet Frames with Several Generators
}

\author{
Jinzhou $\mathrm{Li}^{1, \mathrm{a}^{*}}$ and Zheng Wang ${ }^{2, \mathrm{~b}}$ \\ ${ }^{1}$ College of Mathematical Sciences, Luoyang Normal University, Luoyang 471022, China \\ ${ }^{2}$ School of Arts and Science, Sias International College of Zhengzhou University, Xinzheng 451150, \\ China \\ ajinzhouli1962@163.com, bzhengwang_hd@163.com
}

Keywords: Wavelet; The wave packet frame; Necessary condition

\begin{abstract}
The main goal of this paper is to consider the necessary conditions of wave packet systems to be frames in higher dimensions. The necessary conditions of wave packet frames are established, which include the corresponding results of wavelet analysis and Gabor theory as the special cases. The existing results are generalized to the case of several generators and general lattices.
\end{abstract}

\section{Introduction}

Frames were first introduced by Duffin and Schaeffer[1]. Outside of signal processing, frames did not seem to generate much interest until the outstanding work of Daubechies et al. [2]. Since then, the theory of frames began to be more widely studied. Frames have been used in signal processing, image processing, data compression and sampling theory.

An important example about frame is wavelet frame, which is obtained by translating and dilating a finite family of functions. Wavelets were introduced in the 1980s. They attracted considerable interest from the mathematical community and from members of many diverse disciplines in which wavelets had promising applications. Another most important concrete realization of frame is Gabor frame [3]. They are generated by modulations and translations of a finite family of functions.

In paper [4], authors introduced wave packet systems by applying certain collections of dilations, modulations and translations to a family of functions. In fact, Gabor systems and wavelet systems are special cases of wave packet systems. Wave packet systems have recently been successfully applied to harmonic analysis and operator theory.

The main goal of this paper is to consider the necessary conditions of multiwave packet frames in higher dimensions. We generalize the existing results of the single generator [5] to the case of several generators and general lattices. We establish some necessary conditions for the wave packet frames of the different operator order, which is a generalization of classical wavelet frame and Gabor frame. Of course, our way combines with some techniques in wavelet analysis and time-frequency analysis. Also, we discuss necessary conditions for other wave packet frames with the different operator order. Also, we fuse some ways in wavelet analysis and Gabor theory and we mainly borrow some thoughts in classifying the sufficient conditions of the wavelet frame in existing papers such as [5-8].

\section{Preliminaries}

In this section, some notations and some results which will be used later are introduced.

Let us recall the definition of frame.

Definition 1. Let $H$ be a separable Hilbert space. A sequence $\left\{f_{i}\right\}_{i \in N}$ of elements of $H$ is a frame for $H$ if there exist constants $0<C \leq D<\infty$ such that for all $f \in H$, we have

$$
C\|f\|^{2} \leq \sum_{i \in N}\left\|\left\langle f, f_{i}\right\rangle\right\|^{2} \leq D\|f\|^{2} .
$$


The numbers $C, D$ are called lower and upper frame bounds, respectively (the largest $C$ and the smallest $D$ for which (1) holds are the optimal frame bounds). Those sequences which satisfy only the upper inequality in (1) are called Bessel sequences. A frame is tight if $C=D$. If $C=D=1$, it is called a Parseval frame.

In this paper, we will work with three families of unitary operators on $L^{2}\left(R^{n}\right)$. Let $A \in E_{n}$ and $B, C \in G L_{n}(R)$. The first one consists of the dilation operator $D_{A}: L^{2}\left(R^{n}\right) \rightarrow L^{2}\left(R^{n}\right)$ defined by

$\left(D_{A}\right)(x)=q^{\frac{1}{2}} f(A x)$ with $q=|\operatorname{det} A|$.

The second one consists of all translation operators

$T_{B k}: L^{2}\left(R^{n}\right) \rightarrow L^{2}\left(R^{n}\right), k \in Z^{n}$,

defined by $\left(T_{B k} f\right)(x)=f(x-B k)$. The third one consists of the modulation operator

$E_{C m}: L^{2}\left(R^{n}\right) \rightarrow L^{2}\left(R^{n}\right), m \in Z^{n}$,

defined by $\left(E_{C m} f\right)(x)=e^{2 i \pi C m x} f(x)$.

Let $P \subset Z$ and $Q \subset R^{n}$. Let $S=P \times Q$. Then, we have $S \subset Z \times R^{n}$. Again, let

$\left\{A_{p}: A_{p} \in P\right\} \subset E_{n}$ and $B \in G L_{n}(R)$.

For the functions $\psi^{l} \in L^{2}\left(R^{n}\right), l=1,2, \cdots, L$, we will consider the wave packet system $\Psi$ defined by the following

$$
\Psi=\left\{D_{A_{p}} E_{v} T_{B m} \psi^{l}(x)\right\}_{l=1,2, \cdots, L, m \in Z^{n},(p, v) \in S}
$$

Let $A_{p}=A^{j}(j \in Z), S=Z \times\{0\}$. Then, we obtain the wavelet systems. On the other side, we can get the Gabor systems when the set $\left\{A_{p}: A_{p} \in P\right\}$ only consists of the elementary matrix $E$. This simple observation already suggests that the wave packet systems provide greater flexibility than the wavelet systems or the Gabor systems.

Then, we will give the definitions of wave packet multiwavelet frame and the frame wave packet multiwavelet.

Definition 2. We say that the wave packet system defined $\Psi$ by (5) is a wave packet multiwavelet frame if it is a frame for $L^{2}\left(R^{n}\right)$. Then, the functions $\psi=\left(\psi^{1}, \psi^{2}, \cdots, \psi^{M}\right)$ is called a frame wave packet multiwavelet.

In order to prove the main results to be presented in next section, we need the following lemmas.

Lemma 2.1 Suppose that $\left\{f_{k}\right\}_{k=1}^{+\infty}$ is a family of elements in a Hilbert space $H$ such that there exist constants $0<C \leq D<+\infty$ satisfying (1) for all $f$ belonging to a dense subset $D$ of $H$. Then, the same inequalities (1) are true for all $f \in H$; that is, $\left\{f_{k}\right\}_{k=1}^{+\infty}$ is a frame for $H$.

For proof of Lemma 2.1, people can refer to the book [6].

Therefore, we will consider the following set of functions:

$D=\left\{f \in L^{2}\left(R^{n}\right): \hat{f} \in L^{\infty}\left(R^{n}\right)\right.$ and $\hat{f}$ has compact support in $\left.R^{n},\{0\}\right\}$.

The following result is well known, we can find it in [6].

Lemma 2.2 $D$ is a dense subset of $L^{2}\left(R^{n}\right)$.

The following useful facts can be found in paper [5].

Lemma 2.3 Let $A \in G L_{n}(R), y, z \in R^{n}$ and $f \in L^{2}\left(R^{n}\right)$. Then the following holds:

$$
\begin{aligned}
& \left(T_{y} f\right)^{\hat{n}}=E-y \hat{f},\left(E_{z} f\right)^{\hat{n}}=T_{z} \hat{f},\left(D_{A} f\right)^{\hat{n}}=D_{A^{\sharp}} \hat{f} \text {; } \\
& T_{y} E_{z} f=e^{-2 \pi i z y} E_{z} T_{y} f, \quad D_{A} E_{y} f=E_{A^{*} y} D_{A} f, D_{A} T_{y} f=T_{A^{-1} y} D_{A} f ; \\
& \left(T_{y} E_{z} f\right) \hat{=}=e^{-2 \pi i z y} T_{z} E_{-y} \hat{f} \text {; } \\
& \left(D_{A} T_{y} f\right) \hat{(\xi)}=E_{-A^{\sharp} y} D_{A^{\sharp}} \hat{f}(\xi)=|\operatorname{det} A|^{-\frac{1}{2}} \hat{f}\left(A^{\#} \xi\right) e^{-2 \pi i A^{-1} y \xi} \text {. }
\end{aligned}
$$




\section{Main Results}

In this section, we will consider constructing symmetric Gabor frames with any invertible matrices Motivating by the fundament works [5-8], we will give a necessary condition of wave packet frame $\Psi$ defined by (5) for higher dimension with an arbitrary expansive matrix dilation in the following.

Theorem 3.1 Suppose that wave packet system

$$
\left\{D_{A_{p}} E_{v} T_{B m} \psi^{l}(x)\right\}_{l=1,2, \cdots, L, m \in Z^{n},(p, v) \in S}
$$

defined by (6) is a frame with frame bounds $A_{1}$ and $A_{2}$, then we have

$$
b A_{1} \leq \sum_{l=1}^{L} \sum_{(p, v) \in S}\left|\psi^{l}\left(A_{p}^{\#} \omega-v\right)\right|^{2} \leq b A_{2} \text {, a.e. } \omega,
$$

where $\mathrm{b}=|\operatorname{det} \mathrm{B}|$.

Proof. Because wave packet system $\left\{D_{A_{p}} E_{v} T_{B m} \psi^{l}(x)\right\}_{l=1,2, \cdots, L, m \in Z^{n},(p, v) \in S}$ is a frame with frame bounds $A_{1}$ and $A_{2}$, for all $f \in L^{2}\left(R^{n}\right)$, we have

$$
A_{1}\|f\|^{2} \leq \sum_{l=1}^{L} \sum_{(p, v) \in S} \sum_{m \in Z^{n}}\left|<f, D_{A_{p}} E_{v} T_{B m} \psi^{l}>\right|^{2} \leq A_{2}\|f\|^{2} .
$$

Let $\hat{f} \in C_{c}(R)$ and $\hat{f}$ have compact support.

Let $q_{p}=\left|\operatorname{det} A_{p}\right|$. According to Lemma 2.3 and Plancheral theorem, we have

$$
\begin{aligned}
& \sum_{(p, v) \in S} \sum_{m \in Z^{n}}\left|<f, D_{A_{p}} E_{v} T_{B m} \psi^{l}>\right|^{2}=\sum_{(p, v) \in S} \sum_{m \in Z^{n}}\left|<\mathrm{F} f, \mathrm{~F} D_{A_{p}} E_{v} T_{B m} \psi^{l}>\right|^{2} \\
= & \sum_{(p, v) \in S} \sum_{m \in Z^{n}}\left|<\hat{f}, D_{A_{p}^{\sharp}} T_{v} E_{-B m} \psi^{l}>\right|^{2}=\sum_{p \in P} q_{p}^{-1} \sum_{v \in Q} \sum_{m \in Z^{n}}\left|\int_{R^{n}} \hat{f}(\omega) \overline{\psi^{l}\left(A_{p}^{\sharp} \omega-v\right)} e^{2 \pi i B m\left(A_{p}^{\sharp} \omega-v\right)} d \omega\right|^{2} \\
= & \sum_{p \in P} q_{p} \sum_{v \in Q} \sum_{m \in Z^{n}}\left|\int_{R^{n}} \hat{f}\left(A_{p}^{*}(\omega+v)\right) \overline{\psi^{l}(\omega)} e^{2 \pi i B m \omega} d \omega\right|^{2}
\end{aligned}
$$

where we change variables by $\omega^{\prime}=A_{p}^{\#} \omega-v$ in the last equality.

We assert:

$$
\begin{aligned}
& \sum_{p \in P} q_{p} \sum_{v \in Q} \sum_{m \in Z^{n}}\left|\int_{R^{n}} \hat{f}\left(A^{* j}(\omega+v)\right) \overline{\psi^{l}(\omega)} e^{2 \pi i B m \omega} d \omega\right|^{2} \\
& =\sum_{(p, v) \in S} \frac{q_{p}}{b} \int_{B^{\sharp}\left([0,1]^{n}\right)}\left|\sum_{s \in Z^{n}} \hat{f}\left(A_{p}^{*}\left(\omega+B^{\#} S+v\right)\right) \quad \overline{\psi^{l}}\left(\omega+B^{\sharp} s\right)\right|^{2} d \omega .
\end{aligned}
$$

For fixed $(p, v) \in S$, we have

$$
\begin{aligned}
& \int_{B^{\sharp}\left([0,1]^{n}\right)} \sum_{s \in Z^{n}}\left|\hat{f}\left(A_{p}^{*}\left(\omega+B^{\sharp} s+v\right)\right) \overline{\psi^{l}}\left(\omega+B^{\sharp} s\right)\right| d \omega \\
& =\sum_{s \in Z^{n}} \int_{B^{\sharp}\left([0,1]^{n}\right)}\left|\hat{f}\left(A_{p}^{*}\left(\omega+B^{\sharp} s+v\right)\right) \overline{\psi^{l}}\left(\omega+B^{\sharp} s\right)\right| d \omega \\
& =\sum_{s \in Z^{n}} \int_{B^{\sharp} s+B^{\sharp}\left([0,1]^{n}\right)}\left|\hat{f}\left(A_{p}^{*}(\omega+v)\right) \overline{\psi^{l}}(\omega)\right| d \omega=\int_{R^{n}}\left|\hat{f}\left(A_{p}^{*}(\omega+v)\right) \overline{\psi^{l}}(\omega)\right| d \omega \\
& \leq\left(\int_{R^{n}}\left|\hat{f}\left(A_{p}^{*}(\omega+v)\right)\right|^{2} d \omega\right)^{\frac{1}{2}}\left(\int_{R^{n}}\left|\overline{\psi^{l}}(\omega)\right|^{2} d \omega\right)^{\frac{1}{2}} .
\end{aligned}
$$

where the fourth inequality is obtained by using Cauchy-Schwarz's inequality.

Thus we can define a function $F_{p}: R \rightarrow C$ by

$$
F_{p}(\omega)=\sum_{s \in Z^{n}} \hat{f}\left(A_{p}^{*}\left(\omega+B^{\sharp} s+v\right)\right) \overline{\psi^{l}}\left(\omega+B^{\sharp} s\right) \text {, a.e. } \omega \text {. }
$$

$F_{p}(\omega)$ is $B^{\sharp} T^{n}$-periodic, and the above argument gives that $F_{p}(\omega) \in L^{1}\left(B^{\sharp}[0,1]^{n}\right)$. In fact, we even have $F_{p}(\omega) \in L^{2}\left(B^{\sharp}[0,1]^{n}\right)$. To see this, we first see that 


$$
\left|F_{p}(\omega)\right|^{2} \leq \sum_{s \in Z^{n}}\left|\hat{f}\left(A_{p}^{*}\left(\omega+B^{\#} s+v\right)\right)\right|^{2} \sum_{s \in Z^{n}}\left|\psi^{l}\left(\omega+B^{\sharp} s\right)\right|^{2} .
$$

Since $\hat{f} \in C_{c}(R)$, the function $\omega \rightarrow \sum_{s \in Z^{n}}\left|\hat{f}\left(A_{p}^{*}\left(\omega+B^{\sharp} s+v\right)\right)\right|^{2}$ is bounded. According to above argument, we easily get $F_{p}(x) \in L^{2}\left(B^{\#}[0,1]^{n}\right)$.

Then, according to the definition of $F_{p}(\omega)$, we have

$$
\begin{aligned}
& \int_{R^{n}} \hat{f}\left(A_{p}^{*}(\omega+v)\right) \overline{\psi^{l}(\omega)} e^{2 \pi i B m \omega} d \omega=\sum_{s \in Z^{n}} \int_{B^{\sharp} s+B^{\sharp}\left([0,1]^{n}\right)} \hat{f}\left(A_{p}^{*}(\omega+v)\right) \overline{\psi^{l}}(\omega) e^{2 \pi i B m \omega} d \omega \\
& =\sum_{s \in Z^{n}} \int_{B^{\sharp}\left([0,1]^{n}\right)} \hat{f}\left(A_{p}^{*}\left(\omega+B^{\sharp} s+v\right)\right) \overline{\psi^{l}}\left(\omega+B^{\sharp} s\right) e^{2 \pi i B m \omega} d \omega \\
& =\int_{B^{\sharp}\left([0,1]^{n}\right)}\left(\sum_{s \in Z^{n}} \hat{f}\left(A_{p}^{*}\left(\omega+B^{\sharp} S+v\right)\right) \overline{\psi^{l}}\left(\omega+B^{\sharp} S\right)\right) e^{2 \pi i B m \omega} d \omega \\
& =\int_{B^{\sharp}\left([0,1]^{n}\right)} F_{p}(\omega) e^{2 \pi i B m \omega} d \omega \text {. }
\end{aligned}
$$

Parseval's equality shows that

$$
\sum_{m \in Z^{n}}\left|\int_{B^{\sharp}\left([0,1]^{n}\right)} F_{p}(\omega) e^{2 \pi i B m \omega} d \omega\right|^{2}=\frac{1}{b} \int_{B^{\sharp}\left([0,1]^{n}\right)}\left|F_{p}(\omega)\right|^{2} d \omega ;
$$

Combining (15), (16) and the definition of $F_{p}(\omega)$, we obtain that

$$
\begin{aligned}
& \sum_{m \in Z^{n}}\left|\int_{R^{n}} \hat{f}\left(A_{p}^{*}(\omega+v)\right) \overline{\psi^{l}(\omega)} e^{2 \pi i B m \omega} d \omega\right|^{2} \\
& =\frac{1}{b} \int_{B^{\sharp}\left([0,1]^{n}\right)}\left|\sum_{s \in Z^{n}} \hat{f}\left(A_{p}^{*}\left(\omega+B^{\sharp} s+v\right)\right) \overline{\psi^{l}}\left(\omega+B^{\sharp} s\right)\right|^{2} d \omega .
\end{aligned}
$$

So, we obtain (11). Thus, we complete the assertion.

Choose $\omega_{0} \in R$ to be Lebesgue point of the function $\sum_{(p, v) \in S}\left|\psi^{l}\left(A_{p}^{\sharp} \omega-v\right)\right|^{2}$.

Letting $B(\grave{o})$ denote the ball of radius ò $>0$ about the origin and ò be sufficiently small, define $f_{\grave{o}}$ by $\hat{f}_{\text {蜊 }}(\omega)=\frac{1}{\sqrt{|B(\grave{\mathrm{o}})|}} \chi_{B(}\left(\omega-\omega_{0}\right)$.

Therefore, we obtain

$\left\|f_{\text {蜊 }}{ }^{2}=\right\| \hat{f} \|^{2}=1$.

Thus, we have

$$
\sum_{(p, v) \in S}\left|\psi^{l}\left(A_{p}^{\#} \omega_{0}-v\right)\right|^{2}=\lim _{\grave{o} \rightarrow 0} \int_{\left|\omega-\omega_{0}\right|<\grave{o}} \frac{1}{|B(\grave{o})|} \sum_{(p, v) \in S}\left|\psi^{l}\left(A_{p}^{\#} \omega-v\right)\right|^{2} d \omega .
$$

From the definition of $f,(9),(10)$ and (11), we have

$$
\begin{aligned}
& \int_{\left|\omega-\omega_{0}\right|<\grave{\mathrm{o}}} \frac{1}{|B(\grave{\mathrm{o}})|} \sum_{l=1}^{L} \sum_{(p, v) \in S}\left|\psi^{l}\left(A_{p}^{\sharp} \omega-v\right)\right|^{2} d \omega \\
& =\sum_{l=1}^{L} \sum_{(p, v) \in S} \int_{B^{\sharp}\left([0,1]^{n}\right)}\left|\hat{f}_{\dot{\mathrm{o}}}(\omega)\right|^{2}\left|\psi^{l}\left(A^{\sharp} \omega-v\right)\right|^{2} d \omega \\
& =\sum_{l=1}^{L} \sum_{(p, v) \in S} q_{p} \int_{B^{\sharp}\left([0,1]^{n}\right)}\left|\sum_{s \in Z^{n}} \hat{f}_{\dot{\mathrm{o}}}\left(A_{p}^{*}\left(\omega+B^{\sharp} S+v\right)\right) \psi^{l}\left(\omega+B^{\sharp} S\right)\right|^{2} d \omega \\
& =b \sum_{l=1}^{L} \sum_{(p, v) \in S} \sum_{m \in Z^{n}}\left|<f_{\grave{\mathrm{o}}}, D_{A_{p}} E_{v} T_{B m} \psi^{l}>\right|^{2} \leq b A_{2},
\end{aligned}
$$

Where the third equality is obtained by changing variables $\omega^{\prime}=A_{p}^{*}(\omega+v)$.

Let ò $\rightarrow 0$, using the definition of Lebesgue point, we get 
$\sum_{l=1}^{L} \sum_{(p, v) \in S}\left|\psi^{l}\left(A_{p}^{\#} \omega_{0}-v\right)\right|^{2} \leq b A_{2}$.

According to the definition of Lebesgue point, we obtain

$\sum_{l=1}^{L} \sum_{(p, v) \in S}\left|\psi^{l}\left(A_{p}^{\#} \omega_{0}-v\right)\right|^{2} \geq b A_{1}$.

Comparing with (20) and (21), by changing variables by $\omega=\omega_{0}$, we have (8).

Therefore, we have completed the proof of Theorem 3.1.

Remark 3.1. In particular, let $A$ the elementary matrix $E$ in the Theorem 3.1, then, we obtain the necessary condition of the Gabor frames as the following, which is a generalization of the known result [6] in higher dimensions.

Corollary 3.1 Let $B, C \in G L_{n}(R)$. Suppose that the Gabor system

$\left\{E_{C k} T_{B m} \psi^{l}(x)\right\}_{k, m \in Z^{n}}$

Is a frame with frame bounds $A_{1}$ and $A_{2}$, then

$b A_{1} \leq \sum_{k \in Z^{n}}\left|\psi^{l}(\omega-C k)\right|^{2} \leq b A_{2}$, a.e. $\omega$,

where $\mathrm{b}=|\operatorname{det} \mathrm{B}|$..

On the other side, let

$P=\left\{A^{j}: j \in Z, A \in G L_{n}(R)\right\}$

And $Q=\{0\}$ in the Theorem 3.1, then, we obtain the necessary condition of the wavelet frames as the following, which is a generalization [6] in higher dimensions.

Corollary 3.2 Let $A \in E_{n}, B \in G L_{n}(R)$. Suppose that wavelet system $\left\{D_{A}^{j} T_{B m} \psi(x)\right\}_{j \in Z, m \in Z^{n}}$ is a frame, then $b A_{1} \leq \sum_{j \in Z}\left|\psi^{l}\left(A^{* j} \omega\right)\right|^{2} \leq b A_{2}$, a.e. $\omega$, where $b=|\operatorname{det} B|$.

\section{References}

[1] R. Duffin, A. C. Schaeffer, A class of nonharmonic Fourier series, Trans. Amer. Math. Soc. 72(1952) 341-366

[2] I. Daubechies, A. Groddmann, Y. Mayer, Painless nonorthogonal expansions, J. Math. Phys. 27 (1986) 1271-1283

[3] A. Ron, Z. Shen, Weyl-Heisenberg systems and Riesz bases in $L^{2}\left(R^{d}\right)$, Duke Math. J. 89(1997) 237-282.

[4] D. Labate, G. Weiss, E. Wilson, An approach to the study of wave packet systems, Contemp. Math., Wavelets, Frames and Operator Theory 345 (2004) 215-235

[5] G.C. Wu et al., Necessary conditions and sufficient conditions of the wave packet frames in $L^{2}\left(R^{n}\right)$, Bulletin of the Malaysian Mathematical Sciences Society 37(2014) 1123-1136

[6] K. Grochenig, Foundations of Time-Frequency Analysis Birkhauser Boston, MA, 2001

[7] Say Song Goh, Zhi Yuan Lim, Zuowei Shen, Symmetric and antisymmetric tight wavelet frames Appl. Comput. Harmon. Anal. 20 (2006) 3411-421

[8] Wang L F, Liu Y 2013 Symmetric Gabor frames with several generators in general lattices Int. J. Appl. Math. Stat. 50 (20) 125-131 